\title{
Metodología para analizar la Comprensión Lectora en estudiantes de secundaria
}

\section{Methodology to analyze Reading Comprehension in high school students}

\author{
Midence-Laynes, Mildred Esperanza; Editor académico Prof. Dr. \\ Ängel Sol-Sanchez
}

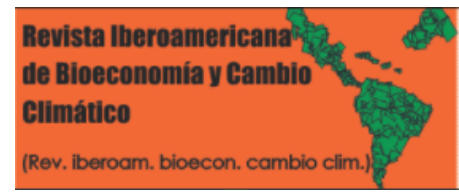

\author{
Mildred Esperanza Midence-Laynes \\ mildred.midence@post.unanleon.edu.ni \\ MINED, Nicaragua \\ Editor académico Prof. Dr. Ängel Sol-Sanchez \\ Colegio de postgraduados, México, Mexico
}

Revista Iberoamericana de Bioeconomía y Cambio Climático

Universidad Nacional Autónoma de Nicaragua, León, Nicaragua ISSN-e: 2410-7980

Periodicidad: Semestral

vol. 2, núm. 4, 2016

czuniga@ct.unanleon.edu.ni

Recepción: 12 Junio 2016

Aprobación: 23 Diciembre 2016

URL: http://portal.amelica.org/ameli/journal/394/3941751012/

DOI: https://doi.org/10.5377/ribcc.v2i1.5706

Autor de correspondencia: mildred.midence@post.unanleon.edu.ni
Resumen: El presente ensayo se centró en analizar los enfoques y metodologías de las estrategias utilizadas en la comprensión lectora al momento de leer cualquier tipo de texto. Se realizó la revisión de la literatura de las metodologías para analizar la compresión de la Lectura en estudiantes de secundaria. Los resultados concluyen en los métodos de análisis para alcanzar buenos resultados en la compresión lectora en los estudiantes de secundaria el estudiante interactúa y resuelve diferentes actividades como: jugando con el verdadero o falsa y la selección de respuestas correctas, al mismo que el estudiantes realice lecturas de textos donde el identifique las ideas principales del texto, criterios a)Cuanto mayor sea el número de semejanzas entre la analogía y la idea a enseñar más efectiva será aquélla. b) Cuanto mayor sea el número de ideas que pueden hacerse familiares con una determinada analogía mayor será su utilidad. c) Cuanto mayor sea la familiaridad y el sentido de la analogía para el alumnado más eficaz será la analogía. d) Puede ser útil emplear más de una analogía, especialmente cuando existen muchas diferencias individuales entre el alumnado. e) Cuando se emplea una analogía debe señalarse con claridad el tipo de estructura a comparar.

Palabras clave: Comprensión Lectora, conocimiento, estrategias, pensar. Abstract.

Abstract: This essay focused on analyzing the approaches and methodologies of the strategies used in reading comprehension when reading any type of text. A review of the literature of the methodologies to analyze reading comprehension in high school students was carried out. The results conclude in the analysis methods to achieve good results in reading comprehension in high school students, the student interacts and solves different activities such as: playing with the true or false and the selection of correct answers, at the same time that the students perform readings. of texts where he identifies the main ideas of the text, criteria a) The greater the number of similarities between the analogy and the idea to be taught, the more effective it will be. b) The greater the number of ideas that can be made familiar with a given analogy, the greater its usefulness. c) The greater the familiarity and the sense of the analogy for the students, the more effective the analogy will be. d) It can be useful to use more than one analogy, especially when there are many individual 
differences between students. e) When an analogy is used, the type of structure to be compared must be clearly indicated.

Keywords: Reading comprehension, knowledge, strategies, thinking.

\section{INTRODUCCIÓN}

En el panorama actual de reforma educativa y perfeccionamiento del profesorado y más concretamente en el marco de técnicas facilitadoras del proceso de aprendizaje llama la atención la cantidad de referencias que se hacen al tema "estrategias de enseñar a pensar". Aunque el profesorado en general se ha preocupado de enseñar a pensar, el momento actual presenta algunas circunstancias nuevas que influyen en la necesidad de formar mejor e informarnos acera de este tema. Los hechos nos demuestran cada día la necesidad de un aprendizaje continuo para poder vivir en unas sociedades que presentan constantemente y de forma rápida cambios culturales, tecnológicos, políticos, científicos, económicos, geográficos, generacionales... Necesitamos una educación que desarrolle la capacidad de pensar y la capacidad de aprender, situando en primer plano la exigencia de un aprendizaje continuo, o el aprender a aprender, como se le denomina otras veces (King y Schneider, 1991). Actualmente existe un movimiento internacional para aumentar y mejorar las estrategias de enseñar a pensar (Nickerson y otros, 1987). Se han generado muchos programas y enfoques diferentes destinados a favorecer el proceso de aprendizaje en todos los currícula y para todos los ciudadanos (Nikerson,1988). Enseñar a pensar es un enfoque que va más allá del objetivo tradicional de los aprendizajes concretos entendidos como cambios en la conducta. Se trata de enseñar a que las personas sean cada vez más conscientes y responsables de sus capacidades, procesos y resultados de aprendizaje siendo así la comprensión lectora la piedra angular puesto que ella permite tener la capacidad de entender lo que se lee, tanto en referencia al significado de las palabras que forman un texto como con respecto a la comprensión global de un escrito . Las investigaciones desarrolladas desde el enfoque de la psicología cognitiva han modificado las concepciones del proceso de enseñanza-aprendizaje (Shuell, 1986). Este nuevo enfoque pone el énfasis en los procesos internos del sujeto que aprende, resaltando el papel activo de la información que reciben y que a su vez suministran, utilizando una amplia variedad de estrategias en el procesamiento de la información, en la adquisición, elaboración, retención, recuperación y utilización de conocimientos, en la toma de decisiones y en la solución de problemas. En la medida que se puede conocer mejor el proceso de enseñanza-aprendizaje también se puede controlar y mejorar.

\section{Desarrollo}

¿Qué es enseñar a pensar?

La capacidad para aprender continuamente tiene mucho que ver con el aprendizaje de estrategias generales del pensamiento que nos permiten conocer, y buscar la información que necesitamos, en un momento dado, para resolver una tarea o solucionar un problema. Esto implica que tan importante es saber cuál es la información que uno tiene como saber la que le falta, en un momento dado, para resolver la tarea o solucionar el problema. Esto también implica que para favorecer un aprendizaje permanente se necesita sobre todo enseñar al sujeto las capacidades que le permitan generar de manera continua la información que va necesitando. Por lo tanto, podemos entender por estrategias de enseñar a pensar el aprendizaje de estrategias

\section{NotAS DE AUTOR}


generales del pensamiento, que son objeto de instrucción explícita (MEC, 1992). Esta instrucción puede y debe realizarse a través de las diferentes áreas del currículum, aunque puede aparecer explícitamente como objetivo en algunas de ellas. Por ejemplo, enseñar a diferenciar las ideas principales de las secundarias en un texto de Lengua; enseñar a planificar un proceso de trabajo, estableciendo una secuencia de operaciones, tiempo y recursos necesarios en Tecnología; enseñar a seleccionar información relevante a partir de centros de documentación de fácil acceso en Ciencias Sociales...etc. Con otras palabras, el aprendizaje de estrategias generales de pensamiento no es asunto exclusivo de la Filosofía o de las Matemáticas, aunque estas áreas contribuyan de manera específica en su desarrollo, sino que pueden desarrollar a través de todas y cada una de las áreas (Ruggiero, 1988; Fischer,1990). En el capítulo siguiente se analizarán los diferentes componentes del proceso de enseñar a pensar.

¿Se puede enseñar a pensar? La respuesta a este interrogante para poder resolver antes la cuestión de la modificabilidad de la inteligencia. Si la inteligencia es o no modificable se inscribe en el viejo marco histórico de las disputas entre genetistas y ambientalistas. Actualmente la cuestión no se plantea en términos disyuntivos "o herencia" "o ambiente", sino en los del grado y forma que presenta su indiscutible interacción. Herencia y medio sí pero ¿cuánto de una y de otra? ¿Cómo interactúan entre sí? (Pinillos, 1982). Son bien conocidas las experiencias de Feuerstein (1985) realizadas con hijos de emigrantes recién llegados a Israel. Este psicólogo sospechó que los malos resultados de esos niños en los test de inteligencia podían deberse fundamentalmente al hándicap sociocultural provocado por el cambio al que fueron sometidos y también quizás, por la de privación cultural que ya traían. Con otras palabras, los tests que se utilizaban para medir la inteligencia no eran socioculturalmente neutrales, y favorecían a los sujetos de la cultura dominante y de niveles socioeconómicos más elevados. Feuerstein trató de aplicar un programa de adiestramiento que compensara en lo posible esos déficits socioculturales. Trató fundamentalmente de "medir" no solamente lo que ya sabían los sujetos sino sobre todo determinó lo que eran capeces de aprender o si se prefiere su "potencial de aprendizaje". Este potencial nos indica las posibilidades de cambio futuro y las posibilidades reales de mejora del sujeto. No mide lo que es sino lo que puede ser a nivel intelectual. Existen pruebas empíricas que ponen de manifiesto la modificabilidad cognitiva. Un requisito fundamental de esta modificabilidad cognitiva es la mediación o mediaciones instrumentales y sociales que usemos en el proceso de aprendizaje. Algunos autores que han estudiado la adquisición del conocimiento (Piaget, 1982) han puesto mucho énfasis en la naturaleza constructiva personal del sujeto y cada vez más se reconoce la importancia de la dimensión social. El aprendizaje y el pensamiento tienen lugar en un contexto social, están influenciados por la cultura y el entorno histórico e institucional en el que se inscriben. En la figura 1 se presenta un modelo representativo del funcionamiento mental de la persona, el papel de la cognición, de la metacognición y de la motivación- afectividad en un contexto cultural, histórico e institucionalmente determinados. Actualmente sabemos que los procesos cognitivos se desarrollan gracias a las interrelaciones sociales que el sujeto realiza en su entorno.

Figura 1.- Un modelo representativo del funcionamiento mental de la persona, el papel de la cognición, de la meta cognición y de la motivación-afectividad en un contexto cultural, histórico e institucionalmente determinado.

Estas capacidades cognitivas se transmiten socialmente y se usan mediatizadas por contextos culturales. Por lo tanto, ese potencial de aprendizaje tiene mucho que ver con "la zona de desarrollo próximo" (ZDP) de la que habla Vigotsky. La ZDP sería la distancia entre el nivel real de desarrollo (determinado por la capacidad de resolver independientemente un problema) y el nivel de desarrollo potencial (determinado a través de la resolución de un problema bajo la guía de un adulto o en colaboración con otro compañero más capaz). El entrenamiento en estrategias de enseñar a pensar se sitúa precisamente en esta ZDP que el profesorado necesita detectar en su aula. Si seguimos el modelo vigotskiano, los ZDP sugiere que la posible intervención del profesorado esté inversamente relacionada con el nivel de desarrollo de la persona en una tarea dada. Cuanto mayor sea el grado de dificultad que experimente el alumnado, más intervenciones directas y ayudas 
del profesorado necesitará. Como señalan algunos autores (Nickerson y otros, 1987) no hay pruebas que demuestren que esas estrategias cognitivas -que se sitúan en la ZDP-surjan automáticamente complejas

Estructuras y procesos cognitivos

\section{MOTIVACION Y AFECTIVIDAD}

Identidad personal motivación expectativas reacciones afectivas evaluación, competencia y control continua planificación, control y evaluación uso de estrategias.

Resultado de la maduración sino que son susceptibles de mejora mediante el entrenamiento. Se puede afirmar que los hechos demuestran que se pueden enseñar estrategias cognitivas que permitan al alumnado hacer un mejor uso de lo que ya conoce y de lo que sabe hacer, de tal manera que se le capacite para buscar nuevas respuestas a nuevos problemas que se le vayan presentando. Dando un paso más, parece que en la medida que el alumnado toma conciencia de la naturaleza y procesos de su pensamiento, tiene más capacidad de conocimiento y control, con lo cual, parece deseable que los diversos componentes de la meta cognición sean objetivo explícito de enseñanza- aprendizaje. Con otras palabras, tanto el profesorado como el alumnado pueden conocer, controlar y mejorar las propias estrategias cognitivas, motivacionales y metacognitivas (García, 1991). La posibilidad de enseñar a pensar se centra en el papel mediador y facilitador que tiene el profesorado en el proceso de aprendizaje del alumnado y se centra también en la dimensión del profesorado como agente cultural, crítico y activo en el entorno sociales en que se desarrolla su profesión (Giroux, 1990). Una última precisión en relación con el enseñar a pensar es la consideración de que no podemos dejar de lado la parte afectiva y motivacional de los sujetos que aprenden. No hay necesariamente una dicotomía entre pensamiento y motivación. De la misma manera que el enseñar a pensar se realiza en un contexto socio-cultural también el enseñar a pensar se desarrolla y requiere una base motivacional y un clima afectivo determinado, como veremos más adelante.

\section{ESTRATEGIAS PARA ENSEÑAR A PENSAR.}

El proceso de aprender a aprender presenta algunas dimensiones importantes que tienen que ver con "la cognición" y "la meta cognición", que pasamos a analizar brevemente. El término "cognición” es genérico y se refiere a procesos cognitivos específicos como atención, percepción, memoria, pensamiento, razonamiento, etc. El término "meta cognición" hace referencia al conocimiento y control de los procesos cognitivos, como veremos seguidamente. El entrenamiento cognitivo pretende desarrollar en el alumnado capacidades, procedimientos o estrategias que le permitan adquirir, elaborar y recuperar información o conocimiento. El entrenamiento meta cognitivo pretende desarrollar en el alumnado el conocimiento sistemático y deliberado de aquellas estrategias cognitivas necesarias para el aprendizaje eficaz, así como la regulación y control de tales estrategias. Un sujeto consciente de sus propios procesos cognitivos es un sujeto más activo, responsable y eficaz frente a los aprendizajes, en definitiva, más capaz de aprender a aprender (Novak y Gowin, 1988). En el estudio del aprendizaje ha dominado durante varias décadas el enfoque conductista que presentaba especial atención a las conductas manifiestas del sujeto que aprende. Este enfoque es el que ha predominado en el sistema educativo español hasta la Ley de Educación en 1970. El aprendizaje se define como cambios relativamente estables en la conducta del sujeto como resultado de la experiencia, y el objetivo consistía en establecer asociaciones entre estímulos y respuestas mediante la práctica. El enfoque cognitivo, por el contrario, pone el énfasis en los procesos internos del sujeto que aprende, resaltando el papel activo y responsable del mismo en su propio proceso de aprendizaje. La perspectiva cognitiva estudia las operaciones, procesos o estrategias que realiza el sujeto cuando aprende, es decir, cuando adquiere, organiza, elabora y recupera conocimientos. Además se pone de relieve las diferencias que muestran los sujetos expertos 
en relación con sujetos noveles, a fin de poder diseñar procedimientos para mejorar los aprendizajes de estos últimos. Si se puede enseñar al alumnado que presenta deficiencias y dificultades en el aprendizaje, procedimientos o estrategias para superarlas, sería inadmisible no hacerlo. Se han propuesto diferentes denominaciones y clasificaciones de estrategias de aprendizaje (Nisbet y Shucksmith, 1987). Vamos a diferenciar en el aprender a aprender tres tipos diferentes de estrategias, según se representan en la Figura 2.

\section{ESTRATEGIAS METACOGNITIVA}

Conocimiento control, planificación, supervisión y evaluación.

El punto clave del aprender a aprender estriba en ofrecer al sujeto herramientas que le ayuden a tomar conciencia de su proceso de aprendizaje y que sea la persona misma quien lo supervise y controle. De ahí que muy frecuentemente se hable en psicología del aprendizaje de favorecer el conocimiento meta cognitivo del sujeto. Cuando hablamos de estrategias de enseñar a pensar nos referimos a este conocimiento meta cognitivo. Podemos precisar ahora un poco más en que consiste. La meta cognición hace referencia a los procesos de pensamiento que la persona tiene acerca de su propio sistema cognitivo (contenidos, procesos, capacidades, limitaciones...) y, por otra parte, a los efectos reguladores que tal conocimiento puede ejercer en su actividad. Según Flavell ( 1981, 1985 ) ( véase la figura 3 ), este conocimiento meta cognitivo estaría formado por tres variables importantes : variables personales o conocimientos de las capacidades y limitaciones cognitivas propias, variables de tarea o conocimiento de las características y dificultades específicas de una tarea determinada y variables de estrategia o conocimiento de las ventajas o inconvenientes de los diferentes procedimientos en la realización de las tareas.

1. VARIABLES PERSONALES

2. •capacidades

3. •limitaciones

1. VARIABLES DE TAREA

2. •características

3. •dificultades

1. VARIABLES DE ESTRATEGIA

2. •ventajas de los diferentes procedimientos en la realización de una misma tarea.

3.- Variables que intervienen en el conocimiento meta cognitivo, según Flavell (1981).

Los conocimientos meta cognitivos relativos a las variables personales son los conocimientos que un sujeto tiene sobre sus propios conocimientos. Por ejemplo, "yo tengo buena memoria para el reconocimiento de caras, lo contrario de lo que me ocurre para la orientación espacial”. Estos conocimientos también pueden hacer referencia a otras personas del entorno, al compararlas entre sí o con uno mismo, por ejemplo "mi madre tiene una capacidad de réplica mejor que mi padre ". Los conocimientos meta cognitivos referidos a las variables de tarea son los conocimientos sobre las características de las tareas, que permiten planificar las empresas cognitivas y distribuir de forma eficaz los recursos disponibles. Por ejemplo, conocer que la lectura de un texto sobre una temática difícil y de la que se tiene poca información exige más tiempo y esfuerzo que si se trata de una lectura más fácil y familiar. Los conocimientos meta cognitivos referidos a las variables de estrategia son los conocimientos que permiten planificar y supervisar las estrategias cognitivas. Por ejemplo, no se lee de la misma forma un texto si sólo queremos hacernos una idea de lo que trata, o si por el contrario nos interesa conocer lo que dice en detalle y además recordarlo para un examen tipo test. Las investigaciones sobre meta cognición pretenden que las personas conozcan mejor (sean conscientes de) sus capacidades y limitaciones, para usar diferentes estrategias en la realización de diferentes tareas. El sujeto pondrá en marcha un tipo de estrategia u otro teniendo en cuenta las diferentes características y dificultades de la tarea determinada y según las propias capacidades y limitaciones personales. De ahí la importancia de desentrañar las múltiples relaciones que existen entre estos tres tipos de variables y la necesidad de favorecer en la persona 
la toma de conciencia de estas relaciones. Si una alumna, por ejemplo, conoce lo que se necesita para efectuar una ejecución eficiente de la clase de Dibujo, entonces puede dar los pasos para satisfacer de modo adecuado las exigencias planteadas por la situación de aprendizaje desarrollada en la clase de Dibujo. Sin embargo, si no es consciente de sus propias limitaciones en tanto que sujeto que aprende o de la complejidad de la tarea en cuestión, difícilmente podemos esperar que adopte acciones pertinentes con el fin de resolver adecuadamente problemas que se le vayan presentando. Dando un paso más en la profundización del conocimiento meta cognitivo se podría afirmar que el disponer de conocimiento apropiado acerca de un determinado ámbito no garantiza su aplicación competente en ese caso. Además el conocimiento específico no requiere conocer cómo y cuándo aplicarlo en Contextos concretos ( Beltrán y otros 1987). Parece oportuno diferenciar como se puede observar el la figura siguiente, dos grandes componentes en la meta cognición: conocimiento del conocer y el control del conocimiento.

Contextos concretos ( Beltrán y otros 1987). Parece oportuno diferenciar como se puede observar el la figura siguiente, dos grandes componentes en la meta cognición: conocimiento del conocer y el control del conocimiento.

El control del conocimiento tiene que ver con el estudio de los procesos y mecanismos de planificación, supervisión y evaluación utilizados por un sujeto activo en situaciones de aprendizaje, razonamiento y resolución de problemas. La capacidad de establecer metas y medios apropiados, de determinar si se está logrando un proceso satisfactorio hacia los objetivos, y de modificar debidamente la propia acción, es otro componente de la meta cognición. La meta cognición entendida como regulación y control de la actividad cognitiva implica la participación activa y responsable del sujeto en los procesos de aprendizaje, es decir, antes, durante y después de realizar al actividad en la planificación, supervisión y evaluación de la misma. Se señala que el control del conocimiento que le llama "control ejecutivo", desempeña un papel de enorme importancia en los procesos intelectuales. Los programas de enseñar a pensar que han logrado mayores niveles de transferencia han incluido una instrucción explícita de estrategias de control. Las estrategias se muestran más eficaces cuando los sujetos comprenden su significado, anticipan su necesidad, supervisan y evalúan lo que se va realizando. En general, la meta cognición se destacan las funciones de supervisión y control de las propias actividades mentales, con el fin de tener información de los estados y procesos cognitivos en curso de realización para modificarlos (Espinosa \& Tamayo 2010).

\section{CONOCIMIENTO DEL CONOCER}

Conocimiento del qué Conocimiento del cómo Conocimiento del cuándo y dónde (aquí entrarían también las variables personales, las variables de tarea y las variables de estrategia).

CONTROL DEL CONOCIMIENTO Planificación y aplicación del conocimiento Supervisión (regulación, seguimiento y comprobación) Evaluación (de las variables personales, las variables de tarea y las variables de estrategia). Su caso. Las actividades de regulación de control serán las siguientes: planificación, supervisión y evaluación.

Planificación. Planificar las actividades cognitivas consiste en prever las actividades cognitivas ante las demandas de la situación, asignando los recursos cognitivos disponibles como atención, concentración, organización de las actividades...etc. Supervisión. Son estrategias meta cognitivas que tratan de comprobar si la actividad se está llevando a cabo según lo planificado, constatando las dificultades que aparecen, las causas de las mismas, las estrategias que se utilizan y su efectividad. Evaluación. La evaluación proporciona al sistema cognitivo un juicio sobre la calidad de los procesos y resultados obtenidos. La evaluación meta cognitiva incluye el conocimiento de las propias capacidades y recursos, las exigencias y objetivos de la tarea, los procesos de realización, y los resultados logrados, así como la introducción de las modificaciones y rectificaciones que se estimen necesarias. Por lo tanto, la capacidad de ser consciente, reflexionar, supervisar y evaluar los procesos cognitivos conlleva extraordinarias posibilidades para la efectividad de los aprendizajes. De ahí que ahora 
podamos comprender mejor una frase que tiene bastante divulgación, en relación con el conocimiento meta cognitivo (Nickerson y otros, 1987):

"Los expertos no sólo saben más, saben que saben más, saben mejor cómo emplear lo que saben, tienen mejor organizado y más fácilmente accesible lo que saben y saben mejor como comprender más todavía”.

Situación de las técnicas de estudio.

Parece oportuno clarificar que las clásicas "técnicas de estudio" habría que situarlas actualmente en el nuevo marco teórico que proporciona la psicología del aprendizaje, como se ha visto precedentemente. Las técnicas de estudio también denominadas técnicas de trabajo intelectual o técnicas instrumentales básicas, constituyen actividades intelectuales de focalización, atención y selección de la información para ser codificada elaborada, retenida, y recuperada por el sujeto. Son, pues, un primer paso en la adquisición, retención y utilización de conocimientos. Podrían agruparse en relación con tres factores: Factores motivacionales. La condición previa para aprender está en que el alumnado quiera hacerlo, está interesado y motivado para ello. Lo mejor sería que la motivación para aprender fuera "intrínseca”, es decir, un interés por conocer y aprender más. Pero también pueden ser oportunos algunos incentivos externos, como el refuerzo verbal del profesorado o de los compañeros/as.

Condiciones externas o ambientales. El aprendizaje requiere unas condiciones ambientales que faciliten la concentración como condiciones de lugar iluminación, ausencia de ruido y distractores en general. También es muy importante la planificación de horarios y tiempos para las diversas materias, con pausas para descansos.

Técnicas de estudio y examen. Encaminadas a comprender, retener y recuperar la información como:

- Lectura global previa, diferenciando partes.

- Identificar las ideas principales y secundarias.

- Subrayar la información más relevante.

- Tomar notas y redactar apuntes.

- Hacer consultas a diccionarios enciclopedias...

- Elaborar esquemas y mapas conceptuales.

- Elaborar resúmenes y trabajos escritos

- Repasar, activar y utilizar el conocimiento adquirido.

- Formularse auto preguntas y elaborar guiones de respuestas.

- Reajustar la exposición y respuestas al tiempo disponible.

Es muy importante resaltar que las técnicas de estudio son ingrediente más del currículo, y por lo tanto un contenido procedimental a enseñar y aprender cuando se trabaja las diferentes áreas y materias curriculares (Selmes, 1888; Hernández y García, 1991). Por lo tanto se pueden considerar como estrategias suplementarias, más que alternativas, en ningún caso excluyente de las estrategias para aprender a aprender que se han presentado precedentemente.

Enseñar a pensar y aprender a pensar.

Todos los sujetos cuando aprenden hacen uso de estrategias. En determinadas ocasiones esas estrategias son apropiadas para lograr los aprendizajes, pero en otras no resultan eficaces. Así mismo, en algunas circunstancias, el sujeto puede ser consciente en mayor o menor grado del funcionamiento de tales estrategias, mientras que en otros momentos pueden operar ajenas al conocimiento o control del sujeto. Las estrategias pueden y deben ser objeto de enseñanza y aprendizaje en un contexto institucional, es decir, se pueden y deben enseñar estrategias efectivas para que el alumnado pueda alcanzar las metas que se propone en su proceso educativo. Pero las estrategias de enseñar a pensar no se aprenden súbita y definitivamente, sino que exigen tiempo y una metodología apropiada. Por otra parte, las estrategias no operan en el vacío, sino que necesitan contenidos específicos para adquirirlas y aplicarlas. Una vez que han adquirido y aplicado se pueden después transferir a otros contenidos y contextos distintos. El curriculum de nuestro sistema educativo establece como contenidos del proceso de enseñanza-aprendizaje: conceptos, procedimientos y actitudes. El término "procedimientos" es sinónimo de estrategias. En general se han desarrollado dos caminos en el entrenamiento 
de estrategias: realizar programas paralelos al curriculum académico del alumnado o incorporar la enseñanza y aprendizaje de las estrategias cuando se trabajan con contenidos curriculares de las diversas áreas o asignaturas, con el fin de facilitar la transferencia. Las dos opciones tienen sus ventajas e inconvenientes que no podemos desarrollar aquí, y por ello pueden ser complementarias, si bien la incorporación adecuada en el desarrollo del curriculum es siempre deseable. Nuestro objetivo principal es ayudar al profesorado para que logre un alumnado activa, efectivo, estratégico, autónomo, cooperativo y responsable, que tenga que depender cada vez menos de la información externa y de las instrucciones del profesorado y que pase a depender más de sus propias capacidades para aprender a través de los que ya sabe, consiguiendo lo que no saben todavía, planificando, supervisando, y evaluando sus propios procesos de aprendizaje.

\section{COMO DESARROLLAR ESTRATEGIAS DE ENSEÑAR A PENSAR.}

El contacto con el profesorado y la observación con la posterior narración de su práctica revelan que, si consideramos el conjunto d las estrategias que promueve el profesorado en el aula, éste concede poca importancia, en general, la manera según la cual se ejecuta o lleva a cabo el aprendizaje de una determinada tarea. El profesorado que quiera enseñar a pensar, sin embargo, tiene que prestar atención al hecho de explicitar los pasos que se dan en el proceso de aprendizaje de una determinada tarea. Para ello tiene que tener en cuenta todos los procesos y mecanismos que influyen en el grupo de alumnado con el que trabaje, tal y como hemos visto precedentemente. Además tratará de ir dejando el control del proceso de aprendizaje en el propio alumnado. Para que se lleve a cabo la enseñanza de estas estrategias parece necesario comenzar por desarrollar y potenciar algunas actitudes.

Potenciar actitudes en el profesorado. El paso previo para enseñar a pensar es ser uno mismo una persona que piensa y reflexiona sobre la práctica profesional (Carr y Kemmis, 1988; Elliott, 1990). Se presentan a continuación, teniendo en cuenta a algunos autores (Nickerson y otros, 1987; Ruggiero, 1988) un conjunto de actitudes que formarían parte del profesorado- reflexivo:

- Una actitud tolerante, respetuosa y valorativa del pluralismo vigente en nuestras sociedades.

- Favorecer un clima emocional y afectivo positivo en el aula.

- Desarrollo de la escucha ante las opiniones de los demás.

- Ofrecer diferentes oportunidades para trabajar técnicas de aprendizaje cooperativo con las cuales el alumnado pueda compartir mejor y contrastar sus conocimientos y vivencias en las situaciones de enseñanzaaprendizaje.

- Actitud abierta al cuestionamiento de las cosas y un sentido vivo para preguntarse por todo.

- Interés en la explicación de las cosas y en la relación de los hechos.

- Actitud positiva hacia la novedad e interés por ampliar las experiencias.

- Desarrollar habilidades que fomenten la producción de ideas.

- Conciencia crítica y reflexión previa. • Mostrar que los argumentos se apoyan en hechos.

- Disposición para modificar el criterio propio cuando las pruebas indican que éste debe modificarse. • Capacidad para cambiar la perspectiva.

- Ecuanimidad ante las diferentes opiniones.

- Comprender que existen diferentes maneras de acercarse a la realidad según los diferentes tipos de personalidad y los distintos contextos culturales.

- Desarrollar en el alumnado tipos de razonamiento diferentes.

- Actitud metacognitiva acerca de cómo resuelve normalmente los problemas como reacciona ante las dificultades y que estrategias específicas usa

- Actitud metacognitiva acerca de los propios campos de resistencia al cambio; cuáles son mis reacciones características hacia la complejidad y la incertidumbre; cuáles son los estereotipos que suelo usar cuando reflexiono; en qué circunstancias tiendo más a la irracionalidad. 
- Ser coherentes en al utilización de los métodos, procedimientos y estrategias para realizar los objetivos previstos.

- Detectar la "zona de desarrollo próximo" (ZDP) del alumnado.

- Desarrollar una autoestima positiva en el alumnado.

- Desarrollar un lenguaje preciso cuando se pretende que también lo haga el alumnado.

- Desarrollar un lenguaje preciso cuando se pretende que también lo haga el alumnado.

La influencia de las actitudes y expectativas del profesorado en el comportamiento del alumnado ha sido demostrada en muchas investigaciones. Estas actitudes se verán reforzadas y potenciadas en la medida que el entorno de aprendizaje del aula invite también a pensar desarrollando con creatividad una distribución de espacios y tiempos en donde se produzca una comunicación efectiva sobre el proceso de aprendizaje.

Implicación de la comunidad educativa.

Es necesario que toda la comunidad educativa se implique en el desarrollo de actitudes que favorezcan el enseñar a pensar. Las estrategias de enseñar a pensar tienen que desarrollarse a través de cada una de las áreas del curriculum, puesto que bastantes trabajos ponen de manifiesto que los procesos de aprendizaje interaccionan con los contenidos de aprendizaje (Nickerson, 1988). Otro aspecto importante a tener en cuenta es la consideración de "grupo- clase" como una comunidad de investigación, tal y como hace años ya proponía Lipman (1976). La puesta en práctica de las estrategias de enseñar a pensar en el centro educativo conlleva preparar al profesorado para reflexionar sobre su práctica cotidiana, de forma que les ayude a pensar sobre su realidad educativa y a orientar-regular su actuación en el aula relacionándola con el marco interactivo del centro (Presseisen, 1991). Se trata de favorecer una comunidad de aprendizaje en al cual se de una potenciación mutua entre el profesorado y el alumnado a la hora de mejorar, regular y controlar el proceso de enseñanza-aprendizaje, no solamente dentro del aula, sino también como agentes transformadores de su entorno socioeducativo (Elosúa, 1992). Sería muy interesante incluir cada vez más técnicas de aprendizaje cooperativo (Slavin, 1990) que potencien el enseñar a pensar. En relación con la implicación de todo el profesorado está también el problema de la transferencia o generalización de las estrategias aprendidas en un momento determinado a otras tareas y situaciones diferentes. La mayor parte de las publicaciones en este campo señalan una interacción entre procesos y contenidos. De ahí la importancia de articular este entrenamiento a través de las diferentes áreas del curriculum. Las estrategias de enseñar a pensar no son un sustitutivo de los contenidos relativos a las diferentes áreas. Precisamente el aprender a aprender tiene que llevarse a cabo en un contexto real: la mejor forma de adquirir estas estrategias es a lo largo de todo el proceso de aprendizaje en las diferentes áreas y contando con la colaboración y compromiso del profesorado. Volveremos sobre este punto al hablar de los métodos.

Métodos de entrenamiento

Una vez que se ha hecho hincapié en la necesidad de desarrollar un tipo de actitudes determinadas y la necesidad de implicar en este cambio de actitudes a toda la comunidad educativa podemos determinar más particularmente algunos de los métodos más utilizados a la hora de favorecer estrategias de enseñar a pensar en el alumnado.

El modelamiento meta cognitivo.

El aprendizaje a través de la observación de buenos modelos sigue siendo útil a la hora de enseñar este tipo de estrategias. Se trataría fundamentalmente de enseñar de manera explícita la forma de reflexionar y de actuar de algunas personas expertas en situaciones parecidas a las que queremos enseñar, de tal manera que se presenten modelos, si pueden ser varios mejor, que ejemplifiquen los pasos seguidos para que el alumnado pueda observar los procesos que normalmente permanecen ocultos a la persona novata. El profesorado en este caso explicará, apoyándose en los modelos, de manera directa por qué y cuándo es importante pensar y actuar como lo hacen los modelos. La presencia de diferentes modelos alternativos puede ayudar a respetar y canalizar en el alumnado los diferentes estilos de aprendizaje.

Moldeamiento. 
Este método consiste en tratar de ir acercándose desde la práctica de los alumnos-as a la actuación que deseamos que vayan modificando. A través de diferentes aproximaciones sucesivas se va moldeando desde la experiencia práctica la forma de pensar y de actuar, comprobando en estas situaciones cómo se va acercando el alunado desde lo que va haciendo a lo que pretendemos que haga o adquiera como resultado final del proceso de moldeamiento.

El análisis y discusión meta cognitiva. Este método trata de identificar y valorar los procesos de pensamiento que subyacen en el aprendizaje de una tarea determinada, buscando que el alumnado sea consciente de los mecanismos propios y de los de los compañeros puestos en juego para resolver la tarea de manera que se puedan mejorar. Lo mismo que cuando el profesorado tiene que enseñar algo se da cuenta de lo que tiene claro y de lo que pensaba que tenía claro pero que en el momento de enseñarlo tiene duda, o si se prefiere, lo mismo que cuando uno tiene que escribir algo se da cuenta de lo que tiene claro y de lo que no, de la misma manera es probable que se produzca una comprensión mayor en el alumnado cuando se le pide que explique, elabore o defienda su posición ante o para otros compañeros (el reto de la explicación y la elaboración de lo que se sabe de una manera nueva). Este método puede tener dos variantes: a) el profesorado propone una actividad o tarea y una vez finalizada pide que los participantes escriban o expongan oralmente el proceso seguido o b) el alumnado distribuido en parejas trabaja cooperativamente unas tareas "pensando en voz alta" otras parejas anotan el proceso seguido para exponerlo después al análisis y discusión de todo el grupo.

El auto interrogación meta cognitiva. La peculiaridad de este método en relación con los anteriores estriba en que favorece un sistema de autorregulación del proceso de aprendizaje, a través de interrogantes que el profesorado se hace antes, durante y después de la realización de una tarea. En la parte de experiencias se presentan los pasos concretos de este método. Se distinguen tres fases en la aplicación de este método: a) el profesorado propone un modelo de interrogación que emplea en varias tareas-ejemplo; b) el alumnado pone a prueba el modelo con varias tareas y c) posteriormente se trata de que el alumnadovaya automatizando el procedimiento para que sea capaz de utilizarlo. Es importante señalar que estos métodos presentan algunas características en común y pueden utilizarse combinados o complementariamente. Las circunstancias concretas favorecerán que en un momento determinado se ponga énfasis en uno u otro método. Se proponen finalmente, siguiendo a algunos autores algunos pasos a tener en cuenta en la enseñanza de este tipo de estrategias, independientemente del método que se seleccione.

Pasos a tener en cuenta:

1. Escoger un método concreto. 2. Regular el grado de dificultad durante la práctica guiada.

2.1Comenzar con el material simple e ir argumentando gradualmente la complejidad de la tarea. 2.2 Completar una parte de la tarea del alumnado. 2.3 Presentar información adicional.

2.4 Anticipar aspectos difíciles de la tarea y errores posibles.

3. Ofrecer contextos diferentes para la práctica del alumnado. 3.1 Presentar la realización de algún ejercicio práctico. 3.2Comprometer al alumnado en una enseñanza recíproca en pequeños grupos.

4 Aumentar la responsabilidad del alumnado o devolver al alumnado la responsabilidad de su propio proceso de aprendizaje. 4.1 Ir disminuyendo progresivamente la información que se haya presentado. $4.2 \mathrm{Ir}$ aumentando gradualmente la complejidad y dificultad del material.

4.3 Disminuir la ayuda pedagógica.

5. Suministrar retroalimentación de la tarea.

6. Ofrecer práctica prolongada y espaciada, facilitando la aplicación a nuevos ejemplos.

Este punto es muy importante si se quiere favorecer la transferencia de lo aprendido, tal y como anteriormente se apuntó. En definitiva, se puede considerar que al enseñanza de estos métodos puede facilitar la puesta en práctica de este tipo de estrategias haciéndolo de manera explícita y ofreciendo el control del proceso de aprendizaje al alumnado. 


\section{ESTRATEGIAS DE ENTRENAMIENTO METACOGNITIVO PARA FAVORECER LA COMPRENSIÓN LECTORA}

Se presenta a continuación una profundización de estrategias meta cognitivas en el ámbito de la comprensión lectora, por ser una tarea muy específica y nuclear en los procesos de enseñanza-aprendizaje. La lectura en general y la comprensión lectora en particular son más una destreza o conjunto de destrezas que un cuerpo de conocimientos, hechos o conceptos. Por esta razón explicitamos que la lectura más que un "contenido" de Lengua es una destreza o habilidad que se pone de manifiesto de forma continua a lo largo de todo el proceso educativo. Leer es una actividad muy compleja, pero como en condiciones normales la aprendemos a una edad temprana, y la practicamos con frecuencia, tendemos a considerarla como una tarea relativamente fácil y simple. Por otra parte, y debido a la práctica, la mayoría de los procesos mentales que están implicados en la actividad de leer se han automatizado, se llevan a cabo con mucha rapidez y resultan inaccesibles a la conciencia reflexiva del sujeto lector. Otros procesos, en cambio, forman parte de la experiencia consciente. Precisamente las estrategias meta cognitivas en la comprensión lectora van a tener mucho que ver con ese ámbito de la experiencia consciente. La actividad lectora implica una interacción muy compleja entre diversos procesos perceptivos, cognitivos y lingüísticos en la persona lectora. A su vez estos procesos están muy mediatizados por la experiencia previa y conocimientos previos de la persona lectora, así como los objetivos de la lectura y las características del texto. Cuando leemos un texto con el objetivo de comprender lo que está escrito, no basta con la decodificación de los signos gráficos o letras escritas (procesos perceptivos visuales) y el reconocimiento de palabras y comprensión de su significado (procesos léxicos). Estos son procesos necesarios pero no suficientes para alcanzar una lectura comprensiva. Es preciso poner en juego conocimientos de tipo sintáctico que ponen en relación las palabras, constituyendo unidades mayores como las oraciones y frases con una estructura determinada (procesos sintácticos). Además el lector tiene que comprender el significado de la oración y el mensaje y el contenido del texto, integrándolo con los conocimientos previos del sujeto (procesos semánticos). A su vez también interviene el contexto situacional y su intencionalidad (procesos pragmáticos). En cualquiera de los procesos pueden presentarse problemas y verse afectada la comprensión del sujeto al enfrentarse con la lectura. El profesorado que conoce los profesos tiene más posibilidades de intervenir adecuadamente para ayudar al lector a resolver las dificultades. Las investigaciones constatan que se dan determinadas diferencias en los procedimientos y destrezas entre los buenos y los malos lectores, y que si se entrena a los sujetos en la práctica de ciertos procedimientos y estrategias se consigue mejorar su nivel de comprensión lectora.

Niveles en la comprensión.

Podemos diferenciar distintos niveles en la comprensión lectora, que irían desde un nivel más superficial a un nivel de mayor profundidad: decodificación, comprensión literal comprensión inferencial y meta comprensión.

Decodificar significa descifrar un código; en este caso se trata de dar un significado a las letras impresas. Se admiten dos procesos decodificadores: uno, consiste en asociar la palabra escrita con el significado disponible en la memoria del sujeto. Es un rápido reconocimiento visual de palabras escritas activando directamente su significado. El otro proceso, que también se denomina "recodificación" implica transformar las letras impresas en sílabas y en sonido para activar así el significado. La comprensión literal consiste en combinar el significado de varias palabras de forma apropiada para formar proposiciones. La comprensión literal se atiene a la información explícitamente reflejada en el texto. La meta comprensión es la conciencia y control que el lector tiene de su proceso de comprensión. Consiste en establecer unas metas para la lectura, comprobar si se están alcanzando y rectificar oportunamente en su caso. Abarca, pues, procesos de planificación, supervisión y evaluación que ya hemos descrito en el capítulo sobre meta cognición. En la lectura están presentes unos objetivos cognitivos, cuya consecución hay que supervisar para introducir rectificaciones si es preciso. La persona que lee se enfrenta con un texto desde unas expectativas y unos 
objetivos, selecciona unos procedimientos o estrategias para alcanzaresos objetivos y comprueba si los logra. Cada uno de estos niveles de comprensión viene constituido, a su vez, por otros componentes y procesos, que han sido objeto de investigación de la psicología cognitiva especialmente durante estas dos últimas décadas. Conocer las diferencias entre los buenos y los malos lectores resulta de gran utilidad precisamente para diseñar los procedimientos de intervención a fin de mejorar la comprensión lectora (Sánchez, 1988). Así los lectores pueden presentar dificultades en los procesos de decodificación, acceso léxico, análisis sintáctico, integración de proposiciones, confección de esquemas y resúmenes, elaboración y asimilación de la información, planificación y control de la comprensión. Se sabe que el buen lector realiza estos procesos más rápida y eficazmente consumiendo menos recursos atencionales, que quedan así disponibles para otras actividades. Las estrategias de intervención por parte del profesorado para ayudar al alumnado que tenga problemas en alguno de estos procesos han de ser diferenciadas.

\title{
Factores en la comprensión.
}

La comprensión lectora es una actividad compleja y gradual, como hemos visto, que puede llegar a diferentes niveles de profundidad dependiendo de diversos factores. Tener en cuenta esos factores es condición obligada si se quiere enseñar-aprender eficazmente. Según se representa la figura 6 el nivel alcanzado en la comprensión está en función de los factores que se presentan a continuación. Las características del texto, su temática y dificultad. Cada tipo de texto presenta una estructura y organización propia. Cuando el alumnado se enfrenta a un texto tiene que tener en cuenta las características del tipo de texto que es. Por ejemplo, es distinto leer un cuento, un informe científico, un fragmento histórico o un artículo de periódico. Los conocimientos previos que tenga el alumnado sobre el tema. El disponer de algunos esquemas en relación con la estructura cognitiva del alumnado para poder activar posteriormente las relaciones conceptuales que tienen, fruto de la propia experiencia, puede facilitar mucho la comprensión y asimilación del mensaje del texto. Las relaciones bien establecidas y amplias entre los diferentes contenidos y unos esquemas cognitivos bien estructurados son condiciones necesarias para una buena comprensión. Todos hemos experimentado lo diferente que resulta leer un texto que trata de una temática familiar frente a otra desconocida.

Figura 4. Factores en la comprensión lectora.

Los objetivos, propósitos y expectativas del alumnado. Al enfrentarse ante un texto la persona que lee va con unos determinados objetivos y expectativas desde los cuales interpreta en mensaje. Por ejemplo, pasar hojas de periódico leyendo los titulares de las noticias más importantes durante unos minutos, es un objetivo distinto a detenerse en los suplementos. Leemos un texto de forma distinta cuando nos van a examinar de su contenido posteriormente, cuando vamos a comprar el objeto que describe o cuando vamos a ponerlo en funcionamiento siguiendo las instrucciones. Los procesos cognitivos, meta cognitivos y lingüísticos que la persona que lee realiza durante la lectura. Estos procesos comprenden atención y concentración en el texto, identificación y reconocimiento de palabras, análisis sintáctico-semántico y pragmático del texto, elaboración y almacenamiento de la memoria a largo plazo, recuperación de la información en la memoria

Conocimientos del lector -declarativos y procedimentales -esquemas y guiones -modelos mentales dominio lingüístico.

\section{COMPRENSIÓN DEL TEXTO}

\author{
Objetivos del lector \\ -metas y planes \\ -Motivaciones y expectativas \\ -Intenciones y propósitos Procesos en el lector
}




\begin{abstract}
-Cognitivos -meta cognitivo
-Lingüísticos

Características del texto

-Tipo, esquema

-Temática

-Dificultad

Operativa y planificación y control de la comprensión que se analiza con más detalle posteriormente.
\end{abstract}

\title{
Estrategias en la comprensión.
}

Un tipo de factores que condicionan la comprensión, según hemos comentado, son los procesos cognitivos y meta cognitivos que el sujeto realiza al leer. Tales procesos requieren distinto grado de conciencia, atención, planificación y control por parte del sujeto. Así, normalmente los componentes fonológicos, léxicos y sintácticos se producen "automáticamente", ajenos a la conciencia del sujeto; mientras que los niveles de comprensión de enunciados e interpretación de textos exigen más recursos cognitivos. Cuando leemos un texto ejecutamos muchas operaciones mentales, y para poder alcanzar el significado del texto resulta necesario que una parte de tales operaciones pasen desapercibidas al sujeto y sin exigirle recursos atencionales. Parece como si determinados niveles de procesamiento operasen de forma "cuasi automática", lo cual posibilitaría, dadas las limitaciones de nuestra capacidad de procesamiento y de memoria operativa, poder dedicar los recursos a los niveles superiores, más conscientes y reflexivos. Las estrategias que presentamos intervienen en los niveles superiores de procesamiento y comprensión que conllevan mayor grado de conciencia y reflexividad. Bien entendido que, para el buen funcionamiento de tales niveles superiores es condición imprescindible que los sistemas de procesamiento inferiores (perceptivos, fonológicos, léxicos, sintácticos) operen adecuadamente. La lectura es una actividad "estratégica". El buen lector pone en juego unos procedimientos o estrategias para obtener un resultado. Tales estrategias o destrezas son susceptibles de ser mejoradas, de convertirse en objeto del proceso de enseñanza-aprendizaje, con el fin de optimizar en los lectores su nivel de comprensión. Si la comprensión es la meta de la actividad lectora es importante que los lectores evalúen si se está logrando. Por ejemplo, un lector experto planifica la actividad lectora, es selectivo al dirigir su atención a los diversos aspectos del texto, supervisa su propia comprensión y afina progresivamente la interpretación del texto.

\section{Estrategias cognitivas}

La comprensión lectora es una compleja actividad cognitiva del procesamiento de información, cuyo objetivo es la comprensión del mensaje escrito. Es posible y además deseable enseñar al lector procedimientos o estrategias para llevar a cabo eficazmente tal actividad. La investigación disponible ha constatado que se puede mejorar la comprensión lectora aprendiendo y practicando determinadas estrategias cognitivas, y por tanto ser objetivo específico del proceso de enseñanza-aprendizaje. Las estrategias cognitivas en comprensión lectora son procedimientos u operaciones mentales que realiza la persona que lee durante el procesamiento de información del texto escrito con el objetivo de comprender su significado. Podemos identificar diversos tipos de estrategias (Englewood 1992).

Estrategias de focalización. Mediante estas estrategias el lector concentra su atención en las informaciones del texto que estima más relevante. Están en función de las características del texto y de los propósitos y expectativas del lector. Por ejemplo, resumir el texto, reconstruir las ideas principales, diferenciar la información esencial de los ejemplos y detalles. Las clásicas “técnicas de estudio” estarían en este tipo, como tomar notas, subrayar, hacer esquemas, etc... 
Estrategias de organización. El lector puede reestructurar de forma distinta el texto a fin de hacerlo más significativo y comprensible. Por ejemplo, reorganizando el texto a partir de determinados criterios como importancia jerárquica de acontecimientos o ideas, ordenamiento según lugar, tiempo, número, duración, etc.; secuencia causa-efecto, inducción-deducción.

Estrategias de resolución de problemas. Procedimientos para resolver los problemas que encuentra durante la lectura, por ejemplo, dificultad para comprender palabras, oraciones, relación entre oraciones, esquema de texto. Las estrategias en estos casos pueden ser, por ejemplo, buscar en el diccionario palabras desconocidas, inferir el significado a partir del contexto, deducir el significado descomponiendo la palabra, releer y parafrasear el texto...

Estrategias de elaboración. Estas estrategias permiten integrar la información del texto con los conocimientos previos del lector, a fin de comprender con más profundidad el significado. Por ejemplo, comentar y valorar el texto, generar imágenes y analogías relacionadas con el contenido, reescribir el texto, formular hipótesis, conclusiones, implicaciones teóricas y prácticas.

\section{Estrategias meta cognitivas en la comprensión lectora.}

Supervisión. En esta fase se trata de comprobar si al actividad se está llevando a cabo según lo planificado, o si se encuentran dificultades, y a qué pueden ser debidas, si las estrategias utilizadas son eficaces y apropiadas para alcanzar el objetivo propuesto. El lector se tiene que auto supervisar al avanzar la lectura. Es preciso que el lector presente los objetivos y el grado de aproximación que está logrando. Además un texto tiene partes diversas y con distinto grado de dificultad, partes principales y partes secundarias. El lector ha de conseguir diferenciarlas, pues cuando el lector no es consciente de las dificultades y obstáculos, no puede seleccionar y poner en acción estrategias para superarlas.

Evaluación. La evaluación hace referencia tanto a los 'procesos que se han desarrollado durante la lectura, como a los resultados de la comprensión lectora. La evaluación de los resultados supone constatar el nivel alcanzado en la comprensión. La evaluación de los procesos incluye toda la actividad lectora: establecimiento de objetivos, grado de consecución de los mismos, dificultades presentadas, estrategias utilizadas, eficacia de las mismas y modificaciones introducidas, resultado de la comprensión. Planificación, supervisión y evaluación no se producen necesariamente en una secuencia mecánica, sino que se da una estrecha interrelación: supervisamos y evaluamos lo que planificamos y planificamos la evaluación. Hemos puesto especial énfasis en el entrenamiento de la persona lectora en relación con estrategias que la ayuden a optimizar la comprensión lectora, pero conviene tener presente que también se puede y debe incidir complementariamente en las características del material de lectura, adecuándolo a las capacidades del lector, también se podría hacer hincapié en la organización del entorno de aprendizaje. La enseñanza- aprendizaje de estrategias cognitivas y meta cognitivas requiere un profesorado con información y formulación en este campo de la cognición y meta cognición; pero también un profesorado con entrenamiento y práctica en el autoconocimiento y análisis de los procesos y estrategias que el profesorado mismo utiliza al leer y sobre todo un profesorado comprometido con su práctica educativa y que sabe reflexionar a partir de la práctica.

\section{ConClusión}

Si queremos suscitar en el alumnado la elaboración de imágenes mentales acerca de lo que está trabajando tenemos que decírselo explícitamente. La utilización de las analogías permite la comprensión de contenidos nuevos a partir de su semejanza con ideas familiares. Por ejemplo, "la analogía del zoom" que se utiliza para explicar cómo la secuenciación de contenidos puede ir de lo más general a lo más particular, permitiendo, a su vez, pasar en un momento determinado, de unos aspectos más específicos a algo más general. De la misma 
manera que con un zoom podemos tener, por ejemplo, una panorámica de la Sierra de Madrid empezando por una toma general y global de aspectos de la Sierra para pasar después a una toma más específica y concreta de alguno de los pueblos de la Sierra, sus calles, sus gentes (Aquí iríamos de lo más general a lo más específico). También sería posible para mostrar laSierra de Madrid comenzar por uno de los pueblos concretos de la misma, para pasar después a los aspectos más globales y generales de dicha sierra. (Aquí iríamos del más específico a lo más general). Con este tipo de analogías se puede llegar a hacer inferencias importantes y descubrir rasgos estructurales en un determinado campo. El papel fundamental de la analogía reside en que cuando las nuevas ideas son difíciles de entender y carecen de sentido son útiles porque proporcionan un contexto de conocimiento organizado que hace que sea significativa esa nueva información- La analogía también puede ayudar la recuperación de experiencias concretas que preparan al sujeto que aprende para entender ideas más abstractas y más complejas. Uno de los problemas con la utilización de las analogías es que una analogía puede entenderse como una equiparación de semejanza parcial entre dos situaciones, pero no todas las semejanzas entre las dos situaciones son relevantes. En este sentido es importante que en la utilización de estrategias analógicas intervengan ciertos procesos que seleccionen aquellos aspectos comunes que son relevantes. Entre los criterios señalados por algunos autores (Clement y Gentner, 1991) señalamos: a) Cuanto mayor sea el número de semejanzas entre la analogía y la idea a enseñar más efectiva será aquélla.b) Cuanto mayor sea el número de ideas que pueden hacerse familiares con una determinada analogía mayor será su utilidad. c) Cuanto mayor sea la familiaridad y el sentido de la analogía para el alumnado más eficaz será la analogía. d) Puede ser útil emplear más de una analogía, especialmente cuando existen muchas diferencias individuales entre el alumnado. e) Cuando se emplea una analogía debe señalarse con claridad el tipo de estructura a comparar entre los análogos.

\section{Referencias bibliográficas}

BiBELTRAN, J. MORALEDA, M. ALCAÑIZ, E.G.; CALLEJA, F.G. Y SANTIUSTE, V. (1987): Psicología de la Educación. Madrid. Eudema.

CARR, W. Y KEMMIS, S. (1988): Teoría crítica de la enseñanza. Barcelona. Martínez Roca.

CLEMENT, C.A. Y GENTNER, D. (1991): “Sistemacity as a selection constraint in analogical mapping”, Cognitive Science, 15,89-132.

ELLIOT, J. (1990): La investigación-acción en educación. Madrid. Morata.

ELOSUA, M.R. (1992): "El aprendizaje significativo desde un enfoque social", Revista de Psicología Universitas Tarraconensis, 2.7-16.

Espinosa, L. A., \& Tamayo, A. M. C. (2010). La enseñanza de estrategias metacognitivas para el mejoramiento de la comprensión lectora. Estado de la cuestión. Pensamiento Psicológico, 5(12).

FEUERSTEIN, R.; JENSEN, M.; HOFFMAN, M.B. Y RAND, Y. (1985): "Instrumental Enrichment, an intervention program for structural cognitive modifiability: Theory and practice", en SEGAL, J. W.; CHIPMAN, S.F. y GLASER, R. (Eds.): Thinking and learning skills. Relating instruction to research.Hillsdale, N.J; Erlbaum, vol. 1

FISHER, R. (1990): Teaching children to think. Oxford. Basil Blackwell

FLAVELL, J.H. (1981): "Metacognition and cognitive monitoring: A new area of cognitive- developmental inquiry", en PARKE, H. (De.) Contemporary Readings in Child Psychology. New York, Mc. Graw Hill. FLAVELL J.H. (1985): Cognitive Development. New York, Prentice-Hall

GARCIA, E. (1991): “El programa de Filosofía para niños y el desarrollo de la metacognición”, Aprender a Pensar. Revista Internacional, 4, 44-65.

GIROUX, H.A. (1990) Los profesores como intelectuales. Hacia una pedagogía crítica del aprendizaje. Barcelona, Paidós/MEC. 
KING, A. y SCHNEIDER, A. (1991): Informe del Consejo al Club de Roma. La primera revolución mundial. Barcelona. Plaza Janés.

LIPMAN, M. (1976): “Philosophy for children”, Metaphilosophy, 7.

NICKERSON, M. (1988): “On improving thinking through instruction”. Review of Research in Education, 15,3-57.

NICKERSON, R.S.; PERKINS, D.N. y SMITH, E.E. (1987): Enseñar a pensar: Aspectos de la aptitud intelectual. Barcelona.

Paidós/MEC. NISBET, J. y SHUCKSMITH, J. (1987): Estrategias de aprendizaje. Madrid, Santillana.

NOVAK, J.D. y GOWIN, D.B. (1988): Aprendiendo a aprender. Barcelona. Martínez Roca.

PIAGET, J. (1982): El nacimiento de la inteligencia en el niño. Madrid. Aguilar.

PINILLOS, J.L. (1982): “La modificación de la inteligencia”,Perspectivas, 1, 1-7.

PRESSEINSEN, B.Z. (1991): "Implementación del pensamiento en el currículum de la escuela", Ponencia presentada en el I Congreso Internacional de Psicología y Educación sobre "Intervención Psicoeducativa”, Madrid, noviembre.

RUGGIERO, V.R. (1988): Teaching Thinking across the curriculum. New York, Harper an Row.

SANCHEZ, E. (1988): “Aprender a leer y leer para aprender: características del escolar con pobre capacidad de comprensión”, Infancia y Aprendizaje, 44, 35-57.

SELMES, I. (1988): La mejora de las habilidades para el estudio. Barcelona. Paidós/MEC.

Paidós/MEC. SHUELL, t.j. (1986): “Cognitive conceptions of learning”, Review of Educational Research, 4, 411-436.

SLAVIN, R.E. (1990): Cooperative Learning: theory, research and practice.

Englewood Cliffs, N.J. Prentice-Hall. SOLE, I. (1992): Estrategias de lectura. Barcelona. Graó. 